\title{
Insight on Effectiveness of Frequently Exercised PQ Classification Techniques
}

\author{
B. Devi Vighneshwari ${ }^{1}$, R. Neela ${ }^{2}$ \\ ${ }^{1}$ Dept of Electrical \& Electronics Engg, the Oxford College of Engineering, Bangalore, India \\ ${ }^{2}$ Dept of Electrical \& Electronics Engg, Annamalai University Chidambaram, India
}

\begin{tabular}{l}
\hline Article Info \\
\hline Article history: \\
Received Apr 6, 2016 \\
Revised Jul 25, 2016 \\
Accepted Aug 10, 2016 \\
\hline Keyword: \\
Energy \\
Power quality \\
Power system \\
PQ classification \\
PQ detection \\
Renewable \\
\hline
\end{tabular}

\begin{abstract}
The growing demands of global consumer market in green energy system have opened the doors for many technologies as well as various sophisticated electrical devices for both commercial and domestic usage. However, with the increasing demands of energy and better quality of services, there is a significant increase in non-linearity in load distribution causing potential effect on the Power Quality (PQ). The harmful effects on PQ are various events e.g. sag, swell, harmonics etc that causes significant amount of system degradation. Therefore, this paper discusses various significant research techniques pertaining to the PQ disturbance classification system introduced by the authors in the past and analyzes its effectiveness scale in terms of research gap. The paper discusses some of the frequently exercised PQ classification techniques from the most relevant literatures in order to have more insights of the techniques.
\end{abstract}

Copyright $(2016$ Institute of Advanced Engineering and Science. All rights reserved.

\section{Corresponding Author:}

B. Devi Vighneshwari,

Asst. Prof: Dept of Electrical \& Electronics Engg.

The Oxford College of Engineering

Bangalore, India

Email:devioxford@gmail.com

\section{INTRODUCTION}

With the usage of modern technologies the consumer market for energy has also underwent a significant revolution in last 5 years. With the evolution of smart-grid based electrical system, it is anticipated that consumers do get an undistorted current as well as voltage in continuous mode. Unfortunately with the large number of saturated market of power electronics have also introduced sophisticated devices and controller system. Introduction of such advanced technologies not only enhances the customer experience but also increase dynamic demands of the customers [1]. One of the important thing to understand is that Power Quality i.e. PQ plays a very important role in global consumer market. PQ bears multiple perceptions for different types of users. For an example consumer utilities consider PQ in terms of reliability of the system whereas device manufacturing firm consider PQ as standard for streamlined and ethical power distribution services [2]. However, the prime objective of PQ for every consumer is to ensure an optimized usage of current, voltage, and fluctuation in frequencies. With the inclusion of various forms of non-linear load on the power distribution network, it gives rise to operational problem of the electrical devices. This results in various problems e.g. swell, sag, under-voltage, over-voltage, harmonics etc [3]. At present, there is also much usage of renewable energy system as well as advanced power transmission technologies. Usage of such methods highly increases the non-linearity in the system as well as give rise to various issues related to regulation of voltage. Due to this, the complexity associated with the planning and operations increases in the network of electricity supply. The system also witnesses significant level of harmonics as well as power sinusoids owing to unexpected alterations in the contingencies in the network, disturbances of load, etc. All these are also called as PQ disturbances events and it works at the cost of 
performance degradation. It also results in permanent and irreversible damage of an electrical device [4]. Section 1 discusses about the intoroduction. Section 2 discusses about the existing system while Section 3 discusses about the research gap. Finally, Section 4 makes summary of the paper.

\subsection{Background}

The term Power Quality or popularly known as PQ is frequently used in power electronic for assessing the superiority of the signal being generated by the system. It can be technically defined as a significant process to control various electromagnetic operations inside a system of power electronics. A system of power electronics are said to deliver quality in power if there is an assurity of undeviated current, voltage, as well as frequency signals in the power systems. When a system experiences unforeseen fluctuation of the current or even a voltage from the usual features, it can lead to serious consequences to the power system. It can even result in system shut down or may produce irreversible damage. The prime reason for such fluctuation is due to uninterrupted alterations of the supply of power. Power Quality can also be defined as a term that can focus on the effectiveness of the power supply with highly synchronized voltage and streamlined current. Although the term PQ sounds more related to power but technically speaking it is more associated with voltage quality rather than electric current or power. It is because power is a term that is related to original energy flow along with the amount of current that is required to maintain the stable load. Table 1 shows the categories of various significant events studied in existing literatures. The prime categories are A. Short duration fluctuation, B. Long duration fluctuation, C. Transient, D. Voltage Imbalance, E. Waveform distortions.

Table 1. Category of Events in Power Quality

\begin{tabular}{|c|c|c|c|c|}
\hline \multicolumn{3}{|c|}{ Category } & Voltage Measurement & Time Period \\
\hline \multirow[t]{8}{*}{ A } & Swell & Momentary & $1.1-1.4 \mathrm{pu}$ & 30 cycles- 3 sec \\
\hline & & Temporary & $1.1-1.2 \mathrm{pu}$ & $3 \mathrm{sec}-1 \mathrm{~min}$ \\
\hline & & Instantaneous & $1.1-1.8 \mathrm{pu}$ & $0.5 \mathrm{sec}-30$ cycles \\
\hline & Sag & Momentary & $0.1-0.9 \mathrm{pu}$ & 30 cycles- 3 sec \\
\hline & & Temporary & $0.1-0.9 \mathrm{pu}$ & $3 \mathrm{sec}-1 \mathrm{~min}$ \\
\hline & & Instantaneous & $0.1-0.9 \mathrm{pu}$ & 0.5 cycles -30 cycles \\
\hline & Interruption & Temporary & $<0.1 \mathrm{pu}$ & $3 \mathrm{sec}-1 \mathrm{~min}$ \\
\hline & & Momentary & $<0.1 \mathrm{pu}$ & 0.5 cycle- $3 \mathrm{sec}$ \\
\hline \multirow[t]{3}{*}{ B } & Under voltage & & $>1 \mathrm{~min}$ & $0.8-0.9 \mathrm{pu}$ \\
\hline & Sustained, Int & & $>1 \mathrm{~min}$ & $0.0 \mathrm{pu}$ \\
\hline & Overvoltage & & $>1 \mathrm{~min}$ & $1.1-1.2 \mathrm{pu}$ \\
\hline \multirow[t]{6}{*}{$\mathrm{C}$} & Oscillatory & High Frequency & $5 \mu \mathrm{sec}$ & $0-4 \mathrm{pu}$ \\
\hline & & Low-Frequency & $0.3-50 \mathrm{msec}$ & $0-4 \mathrm{pu}$ \\
\hline & & Medium-Frequency & $20 \mu \mathrm{sec}$ & $0-8 \mathrm{pu}$ \\
\hline & Impulsive & Millisecond & $>1 \mathrm{msec}$ & - \\
\hline & & Microsecond & $50-1 \mathrm{msec}$ & - \\
\hline & & Nanosecond & $<50 \mathrm{nsec}$ & - \\
\hline $\mathrm{D}$ & Voltage imbal & & $0.5-2 \%$ & Steady State \\
\hline \multirow[t]{3}{*}{$\mathrm{E}$} & Noise & & - & Steady State \\
\hline & Notching & & - & Steady State \\
\hline & Harmonics & & - & Steady State \\
\hline
\end{tabular}

Studies towards PQ is not new and it dates back more than a decade years back; however, there is an increasing interest on this topic owing to various significant reasons. At present time, the modern devices of electricity use microcontrollers in order to meet performance of application. But it also results in various critical degradation towards quality of power. Extensive usage of sophisticated (or hybrid) capacitors or complex motor drives frequently encounters break downs with increasing load of power. It also results in system malfunction that seriously degrades the power quality. Moreover, the present era of consumer market uses grid as the prime basis of power distribution system that has higher interconnected sub-systems. Hence, a smaller amount of power system degradation will cost the entire grid system to bear the cost of inferior performance with respect to power efficiency and stability. With the usage of the modern day electrical system, various problems surfaces e.g. flickering, fluctuating voltages, distortions in waveforms etc. Hence, the best way to solve such issues of power quality is to perform identification of the power signals followed by classifying good to bad signals. Hence, it is important to investigate effectively about the PQ disturbances for significant understanding the problems and thereby rectifying it. There has been an extensive amount of investigation towards this problem. The significant processes adopted in enhancing the quality of the power are segmentation, feature extraction, artificial intelligence, etc. In this process, segmentation and feature 
extraction is quite common in all. Basically, segmentation, feature extraction are the essential steps to design any classifier system for classifying PQ events. The standard PQ classifier design is shown in Figure 1 [5].

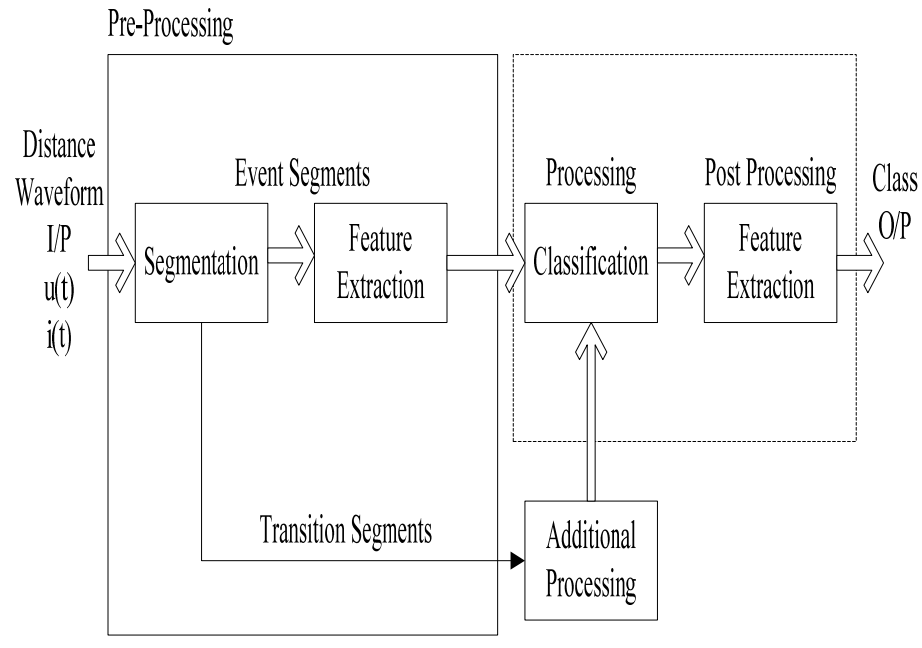

Figure 1. Standard PQ Classifier Design

The input to this classifier design is a waveform with disturbances, which is passed on to block of segmentation that essentially categorize the data in the form of static and non-station section. A significant amount of feature is extracted from the waveforms (with event). A large amount of information can be extracted from the static signal just to carry out comparative analysis of different forms of PQ disturbances. The point-to-point is the frequently adopted technique to compare the statistical values of the disturbed waveforms and pure signal. Methods e.g. Regression framework, Kalman Filter, Short-Termed Fourier Transforms are frequently adopted. Similarly, feature extraction is responsible for identifying and separating significant features from the signal in order to understand the potential signal. The job of classifier is to categorize or classify various types of power quality disturbances. At present, there are two forms of classifier viz. classifier based on statistical approach and classifier based on deterministic approach. Statistical approach [6] is used in a situation of availability for massive dataset while deterministic approach [7] is used for limited size of dataset. A good example of classifier based on statistical approach will be Support Vector Machine and Neural Network [8], while example for classifier for deterministic approach will be fuzzy logic and rule-based expert system [9]. The final stage of PQ classification technique is decision making. The next section discusses about the essential techniques used for enhancing the power quality classification processes. The process of feature extraction plays a critical role in PQ classification process as technical meaning of feature is disturbances in PQ event classification. Therefore, extracted feature gives the direct information about the identification of the disturbances and hence is used for classifying various events of PQ. Figure 2 gives pictorial representation of standard classification of existing process of feature extraction in PQ.

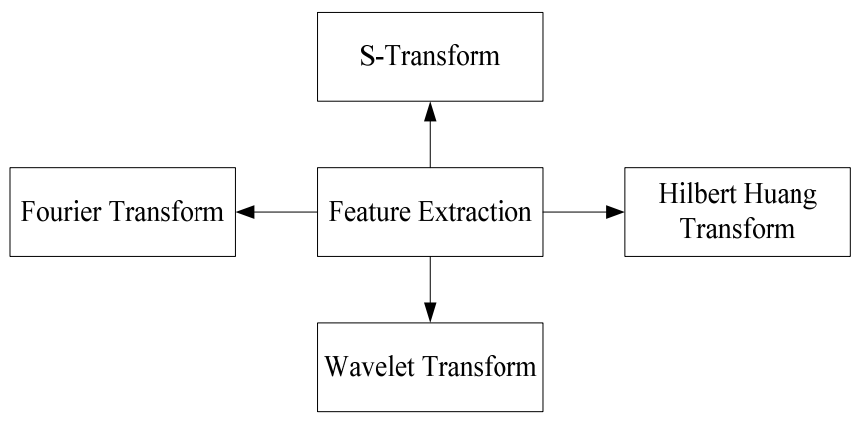

Figure 2. Standard Classification of Feature Extraction in PQ Classification 
The existing methods of classification of feature are:

i. Fourier Transform

It is highly suitable for static signal and when there is a need to capture spectrum at particular level of frequency. One of the specific forms of Fourier Transform is also called as Short-Term Fourier Transform that splits the signal into diminutive static fragments. Hence, Short-Term Fourier Transform evaluates the sinusoidal frequency as well as phase contents of local signal fragments. Using moving window, Short-Term Fourier Transform captures information about signal frames. It is suitable technique for dynamic and nonstatic signal over constant size of window.

\section{ii. Wavelet Transform}

This method performs dilation of the signal prototype function and thereby performs signal decompositions on various levels. It uses group theory representation as well as square-integral function to provide definitive information of frequency and time for a particular signal. Discrete Wavelet Transform and Continuous Wavelet transform are the two frequently used techniques in PQ classification process.

\section{iii. S-Transform}

This form of technique is designed by integrating the features of wavelet transforms and Short-Term Fourier Transform. It uses time-series analysis for assessing the real and imaginary contents from the spectra. An interesting fact about the S-transform is the usage of a typical pattern directly represents a specific event of disturbance in PQ. This technique is also found to be integrated with various forms of other techniques (e.g. artificial intelligence) to enhance its functionality.

\section{iv. Hilbert Huang Transform}

This process performs decomposition of the signal to generate the knowledge-based information about the amplitude and frequency of a signal. The technique makes use of empirical mode decomposition and arranges the frequency in descending order. The decomposed signal is subjected to Hilbert transform to get more precise information about the event classification in power quality.

Table 2. Effectiveness of PQ Classification

\begin{tabular}{|c|c|c|c|c|c|}
\hline \multirow{2}{*}{ No } & \multirow{2}{*}{ PQ event } & \multicolumn{4}{|c|}{ Existing Techniques } \\
\hline & & FFT & DFT & S-T & HHT \\
\hline 1 & Sag & 95 & 98.67 & 100 & 100100 \\
\hline 2 & Swell & 98 & 99.33 & 100 & 95 \\
\hline 3 & Harmonic & 100 & 99.33 & 100 & 100 \\
\hline 4 & Flicker & 89 & 98.67 & 100 & 100 \\
\hline 5 & Notch & - & 97.33 & 83 & 95 \\
\hline 6 & Spike & - & - & 77 & 98 \\
\hline 7 & Transient & 100 & 98.67 & 100 & 98 \\
\hline
\end{tabular}

Table 2 highlights the effectiveness of the existing PQ detection techniques in terms of percentage, where it can be seen that S-transforms was found to posse's better performance than Hilbert Huang Transform (HHT). The significant advantage of usage of Short-Term Fourier Transform is that it can be used for static signals and it is quite easier to implement. The benefit of using HHT is its capability of capturing features from waveforms of distorted types. It can also produce quadrature signal that can directly be used for evaluating phase and amplitude of a signal. The benefit of using S-transform is its inherent charecteristics of simple conversion from time to frequency domain and then to Fourier Frequency Transform (FFT). Similarly, Wavelet Transform can provide precise representation of frequency and time for furnishing better resolution of PQ curves. Likewise, the requirement of higher power quality can also be achieved using Gabor Transform. It has the potential capability of producing outcome with maximal signal-to-noise ratio with superior resolution of the signals.

Hence, it can be seen that there is an existence of various standard methods responsible for carrying out feature extractions of a signal. The next section discusses about other frequently exercised techniques to enhance the PQ disturbance detection and classification process.

\subsection{The Problem}

The modern consumer market of power distribution has witnessed a paradigm shift of consumer towards the new service providers in case the PQ is not found satisfactory by their existing service providers. It becomes therefore highly mandatory for the service provider to furnish higher degree of PQ in their 
services. The issues for PQ classification is basically two types viz. i) events and ii) steady state fluctuations. Events are estimated by abnormality in the current and voltage. Steady-state fluctuation is estimated by the measure by which the current as well as voltage can differ from the standard value along with distortions. It also considers amount of disturbance between the phases (harmonic, voltage variation, distortion).

\subsection{Proposed Study}

Therefore, it is very much important that significant events of PQ classification should be surveilled effectively. However, there has been a volume of research work being already carried out within the research community. It is found that existing power systems are highly complex and massive, which makes the data analysis most complex and unreliable. Therefore, even with presence of massive research archives, standard work towards PQ event classification still misses out from the literatures. There is a need of evolving up with a cost effective solution towards reliable and effective analysis of event detection in PQ study and investigates the underlying operations responsible for system performance enhancement. The process of feature extraction and classification are the most critical phases which requires more amounts of attentions to understand more about disturbances in PQ. Basically, PQ is assessed under various conditions, which has been less investigated. Therefore, this paper is an attempt to understand the most frequently practiced techniques for PQ classification in power electronics

\section{REVIEWING EXISTING TECHNIQUES}

This section discusses about the various existing techniques that was introduced for the purpose of carrying out classification of PQ disturbances in power electronics.

\subsection{Artificial Neural Network}

The usage of artificial neural network is played a huge contributory role in various system of computing world e.g. approximation of function, pattern recognition, clustering, optimization etc. Out of all these potentials, approximation of functions as well as classification charecteristics has been used in investigating PQ classification process.

One of the standard works of PQ classification was found to be carried out by Monedero et al. [10]. The approach considers signal as an input which is subjected to pre-processing followed by neural network classifier. The technique (Figure 3) has the capability to classify electrical disturbance in the form of voltage, frequency, and harmonics. The accuracy in detection of disturbance was found to be $98.73 \%$ over events of sag, swell, under-voltage, and over-voltage.

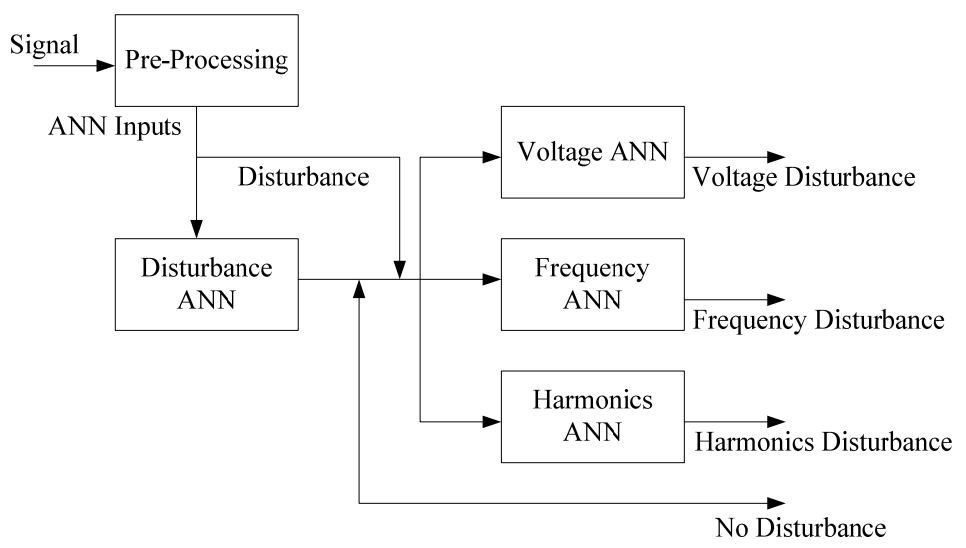

Figure 3 Approaches of Monedero et al. [10]

Dastfan and Zadeh [11] have used S-transform as well as neural network. The study has nearly similar approach with difference of applying S-transform after preprocessing the signal. The technique has also used backpropagation neural network algorithm considering the case study of 3 types of events e.g. i) harmonic and sag, ii) sag and flicker, and iii) flicker and harmonic. The experimentation was carried out over IEEE 34 bus standard. The overall accuracy of detection was found to be around 97-98\% for 1000-2000 epochs respectively. Similar line of investigation was also carried out by Mishra et al. [12], who have used Stransform and neural network for performing classification of PQ considering 11 cases of events. The authors 
have used feed-forward algorithm. The outcome of the study was assessed using accuracy parameters for 3 and 4 features that were found to be around $95-97 \%$.

Viskadouros et al. [13] have used neural network along with wavelet-based approach in order to identify the PQ events. The approach is nearly similar to that presented 3 years back by Mishra et al. [12], Similar version of study along with usage of empirical mode decomposition was seen in the study of Manjula and Sarma [14]. The authors have used probabilistic neural network. Memon et al. [15] have developed a feedforward training mechanism for classification purpose. The prime intention of the study was to minimize the training efforts and correctly identify the source of power disturbances. Although, the work is more inclined towards using wavelet transforms but neural network has assisted to enhance the computational performance of the study. The essential study contribution was to use radial basis function and multi-layered perceptron. The outcome of the study was evaluated with respect to static and non-static signals to find accuracy of $97.45 \%$ in detection rate.

Most recently, the work carried out by Rodriguez et al. [16] have used feedforward neural network for identification and classification of multiple forms of PQ disturbances. The study has also implemented adaptive linear network for estimating harmonics and its distortions. The assessment of the study was carried out on real-time hardware with accuracy of $90 \%$. The study has also performed a comparative analysis with 6 existing techniques (Fuzzy C means, Kalman filter, S-transform with neural network, S-transform with binary feature matrix, S-transform with modular neural network, and fuzzy art map consider 17 cases of an event. Jasper et al. [17] have used back propagation technique for enhancing the power quality of a shunt inverter. The authors have attempted to overcome the issues of conventional pulse wide modulation using neural network. Sundaram [18] have used the similar concept discussed by Rodriguez et al. [16]. Although the accuracy of $97 \%$ is achieved but the paper significant lacks justification and evidence of its outcomes as compared to original version of Rodriguez et al. [16], which has mathematical and empirical evidence behind its outcome. Similar category of study was also carried out by Win et al. [19].

\subsection{Support Vector Machine}

Support vector machine is basically a supervised learning technique that applies statistical learning mechanism. It is a precise technique for carrying for both linear and non-linear classification as well as regression. In the line of research work toward PQ classification, the study conducted by Shakya and Singh [20] has been found to incorporate support vector machine for identifying the problematic area of classification. The study has also used fuzzy classifier. Figure 4 showcases the approach of Shakya and Singh [20], which is also found to be common approach in using artificial intelligence.

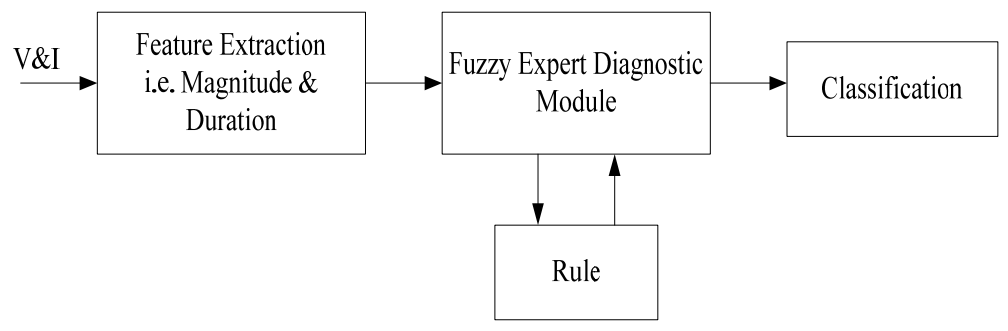

Figure 4. Approaches of Shakya and Singh [20]

Lin et al. [21] have also applied using integral modelling of wavelets with support vector machine in order to carry out classification. The outcome shows the presented system to consume less than 10 seconds of training time. Kocaman et al. [22] have used joint implementation of wavelet based support vector machine. The author have used discrete wavelet transform and performed the classification using support vector machine. Similar line of research has also been carried out by Milchevski et al. [23]. Using the scheme of treebased support vector machine (Figure 5), the author have classified various PQ disturbance events (normal, swell, sag, outage, harmonic, etc) 


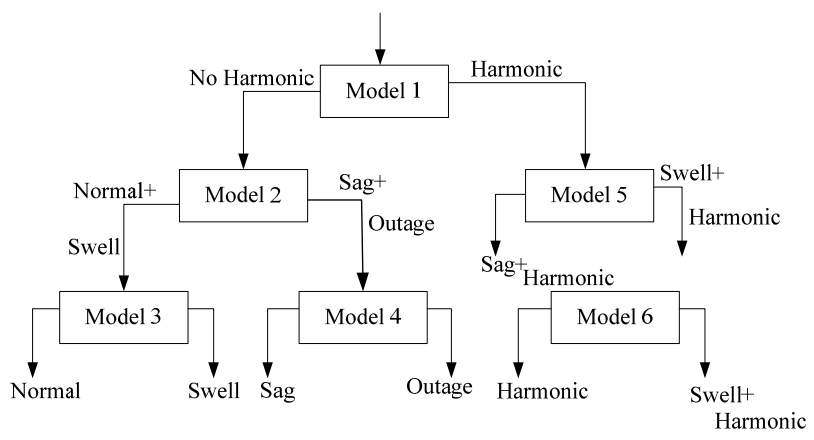

Figure 5. Approaches of Milchevski et al. [23]

Usage of decision tree and support vector machine was also seen in the study of Ray et al. [24]. The authors have used a hardware-based approach considering wind energy system. The outcome of the study was found to posses $99 \%$ of the accuracy in detection of PQ disturbance events. The recent work carried out by Naderian and Salemnia [25], where the author have jointly used support vector machine with Gabor transforms. The outcome of the study was assessed using mean absolute error.

\subsection{Wavelet-based Techniques}

It is found that $70 \%$ of the technique widely used for analysis f PQ classification process is carried out using wavelet-based transforms. He et al. [26] have used self organizing learning array with wavelet transform for performing PQ classification. However, wavelets are mainly preferred for performing feature extraction. The author has performed simulation on 7 events of PQ disturbances with accomplishing accuracy of 94\%. Suja and Jerome [27] have applied wavelets along with neural network for classification of PQ disturbances. Usage of wavelet transform was also seen in significant work of Panigrahi et al. [28] considering 11 case study of PQ disturbances. The authors have used S-transform and neural network. Shareef and Mohamed [29] presented a technique to analysis PQ disturbance using image processing techniques. Nath and Mishra [30] have used wavelets to study the exclusive cases of voltage sag. Eristi and Demir [31] have used logistic tree along with wavelets for carrying our extraction of feature. Roy and Nath [32] have also used wavelets for classification of PQ disturbances along with neural network. The outcome was found to achieve around $95 \%$ in detection of PQ disturbances considering 11 cases of events.

\subsection{Swarm Intelligence}

Swarm intelligence has also seemed to play a vital role in optimization principle. Using concepts of ant colony optimization and particle swarm optimization, some of the recent research work has attempted to perform PQ classification. Nayak and Dash [33] have used particle swarm optimization for enhancing the operation of the cluster centers along with fuzzy approach. The study has also modified discrete S-transform for feature extraction along with k-means clustering. Biswal et al. [34] have adopted the use of ant colony optimization for classifying the PQ. The study has also used fuzzy c-means approach for evolving up with decision tree. The enhancement of the patterns is done using ant colony optimization. The study carried out by Parizi et al. [35] has also used particle swarm optimization along with the S-transform for classifying PQ disturbances. The outcome is recorded with accuracy of 92-97\%. Similar line of study was also carried out by Majhi et al. [36]. Study carried out by Kumarasabapathy and Manoharan [37] have used ant colony optimization along with fuzzy logic for addressing the degraded problems of power quality. The study have designed a new fuzzy controller and performed a simulation study on Matlab. Usage of fuzzy inference system was dominant in the study of Nayak [33] too.

\subsection{Other Classification Techniques}

The other frequently used classification technique uses Expert's system. Tianrui and Sen [38] have presented a technique to automate the power quality controlling mechanism. Jiapei et al. [39] have presented a new inverter system with better controlling capability to ensure power quality. Guo et al. [40] have developed a technique to evaluate power quality using statistical correlation-based technique. The study conducted by Reaz et al. [41] has adopted a unique usage of integrating fuzzy logic, discrete wavelet transform, and neural network. The study outcome was found to posses $98.19 \%$ of accuracy in the detection. Huang and Lin [42] have used maximum likelihood method for developing a classification technique. Most recently Goes et al. [43] have used data mining process to perform classification of the power quality disturbances. 
Table 3 showcases the scale of effectiveness of the existing approaches used for PQ classification. The outcome shows SVM is the most preferred approach followed by Artificial Neural Network. However, fuzzy logic and swarm intelligence based approach still lags behind in terms of classification.

Table 3. Effectiveness of other Classification Techniques

\begin{tabular}{lcccc}
\hline \multicolumn{1}{c}{ Factor } & \multicolumn{4}{c}{ Other techniques } \\
& SVM & ANN & FL & SI \\
\hline Datamining & $* * *$ & $* * *$ & $* *$ & $*$ \\
Knowledge Representation & $*$ & $* * * * *$ & $* * *$ & $* * *$ \\
Sustaining Uncertainty & $* * * *$ & $* * *$ & $* * *$ & $* * *$ \\
Adaptability & $* * *$ & $* * *$ & $* *$ & $* * *$ \\
Generalized Performance & $* * * * *$ & $* * *$ & $*$ & $*$ \\
Learning Ability & $* * * *$ & $* * * *$ & $*$ & $* * *$ \\
\hline
\end{tabular}

\section{RESEARCH GAP}

This section discusses about the significant points that are found to map in terms of research gap. The views briefed in this section are derived from prior sections. Hence, the prominent research gaps explored are:

\subsection{Inherent Issues in Transform Techniques}

The study found transform-based techniques e.g. FFT, S-Transforms, Short-Term Fourier Transform etc have frequently being used. But, Unfortunately, FFT-based approaches are incapable of processing signal with large variation. Short-Term Fourier Transform cannot analyze non-static signals. The usage of HHT is restrictive to only narrow band conditions. S-transforms cannot cater up the real-time requirements and wavelet-based approach is potentially impacted by the level of noise. Even Gabor-based transform approaches are strongly associated with high computational complexities. Such issues of transform-based classification scheme are not found to be addressed in any existing studies.

\subsection{Problems Associated with ANN-based Approach}

All the approaches based on neural network are highly dependent on the size of training data. More the training data more is the level of accuracy. Although ANN could offer better knowledge representation but its generalized performance pattern is not satisfactory. There is a huge trade-off between accuracy and training time, which is still unsolved in the existing system. Another bigger problem is data heterogeneity, which cannot be effectively handled by ANN.

\subsection{Fewer Standard Outcomes}

A robust evaluation of outcomes assists to understand the effective work till date. Our investigation shows that $95 \%$ of the studies conducted till date in PQ classification are not benchmarked. Few to name are study of Monedero et al. [10], Dastfan and Zadeh [11], Mishra et al. [12], Manjula and Sarma [14], Lin et al. [21], Kocaman et al. [22], Milchevski et al. [23], Naderian and Salemnia [25], Suja and Jerome [27], Nath and Mishra [30], Nayak and Dash [33], Biswal et al. [34] etc.

\subsection{Less Novelty in Approaches}

Our investigation found that $45 \%$ of the existing studies are slight enhancement of prior studies. Unfortunately, the slight enhancement has not yield any significant alterations in the outcomes. We found that study conducted by Mishra et al. [12] has actually been replicated by other researchers e.g. Viskadouros et al. [13], Manjula and Sarma [14]. Win et al. [19], Panigrahi et al. [28], Roy and Nath [31] etc. The study conducted by Sundaram [18] is similar to Rodriguez et al. [16]. Similarly, study conducted towards usage of support vector machine has similar work e.g. Kocaman et al. [22] and Milchevski et al. [23]. Likewise, Study conducted by Parizi et al. [44], Majhi et al. [36] have originally used by Nayak and Dash [33].

Hence, these are the significant research gaps towards the study of PQ classification techniques. Hence, the above mentioned issues should be addressed effectively in future.

\section{CONCLUSION \& FUTURE WORK}

From the discussion of Power Quality (PQ) theory and existing research work, it can be seen that PQ disturbances are more or less a direct representation of non-static signals. In the past, the most frequently adopted techniques are artificial intelligence, wavelet-based approach, Swarm intelligence-based approach etc. We found that almost every technique has got advantages as well as potential limitations. The paper has also 
discussed the research gap briefly. From the entire investigation, it can be only said that there should be more analysis on joint mechanism.

Our future work will be focused on developing a novel algorithm for PQ classification in order to enhance the online monitoring system. We will initiate our investigation by implementing the enhanced version of wavelet and neural network-based approach. The prime reason behind this will be to wavelet and neural network-based approach are most frequently adopted technique and thereby comparative analysis becomes easier and less complicated to initiate with. Our future direction will be also towards designing a novel classifier based on enhanced neural network. The work is anticipated to have lesser dependency of higher accuracy on size of training database. Our work will be towards both single and multiple forms of disturbances in PQ events. Our final destination will be to develop a fuzzy-classifier with enhanced multi level classification approach.

\section{REFERENCES}

[1] C. P. Verhoef, et al., "Customer experience creation: Determinants, dynamics and management strategies," Journal of retailing, vol/issue: 85(1), pp. 31-41, 2009.

[2] J. B. Karmacharya, "Good Manufacturing Practices (GMP) for Medicinal Products," 2014.

[3] R. Jangid and P. A. K. Parkh, "Reducing the Voltage Sag and Swell Problem in Distribution System Using Dynamic Voltage Restorer with PI Controller," Retrived 22nd Oct, 2015.

[4] T. Fang, et al., "Process for electrically connecting electrical devices using a conductive anisotropic material," U.S. Patent, 1997.

[5] D. Saxena, et al., "Power quality event classification: an overview and key issues," International Journal of Engineering, Science and Technology, vol/issue: 2(3), pp. 186-199, 2010.

[6] C. Thurau, et al., "Deterministic CUR for Improved Large-Scale Data Analysis: An Empirical Study," in SDM, pp. 684-695, 2012.

[7] J. Solomon and B. Rock, "Imaging spectrometry for earth remote sensing," Science, vol/issue: 228(4704), pp. $1147-1152,1985$

[8] V. Kecman, "Learning and soft computing: support vector machines," neural networks, and fuzzy logic models. MIT press, 2001.

[9] C. C. Lee, "Fuzzy logic in control systems: fuzzy logic controller II," Systems, Man and Cybernetics, IEEE Transactions, vol/issue: 20(2), pp. 419-435, 1990.

[10] Monedero, et al., "VICARED: A neural network based system for the detection of electrical disturbances in real time," in Advances in Natural Computation, Springer Berlin Heidelberg, pp. 147-154, 2005.

[11] Dastfan and A. S. Zadeh, "Intelligent Power Quality Monitoring by using S-Transform and Neural Network," Retrieved, 10th November, 2015.

[12] S. Mishra, et al., "Detection and classification of power quality disturbances using S-transform and probabilistic neural network," Power Delivery, IEEE Transactions, vol/issue: 23(1), pp. 280-287, 2008.

[13] G. Viskadouros, et al., "Power Quality Event Recognition and Classification Using a Wavelet-Based Neural Network," Int. J. of Sustainable Water and Environmental Systems, vol/issue: 3(1), pp. 45-52, 2011.

[14] M. Manjula and A. V. R. S. Sarma, "Assessment of power quality events by empirical mode decomposition based neural network," in Proceeding of the World Congress on Engineering, pp. 4-6, 2012.

[15] A. P. Memon, et al., "Time-Frequency and Artificial Neural Network Applications and Analysis for Electrical System Power Quality Disturbances in MATLAB," International Journal of Innovative Technology and Exploring Engineering (IJITEE), www. ijitee. org 3, Retrieved, 10th Nov, 2015.

[16] M. V. Rodriguez, et al., "Detection and classification of single and combined power quality disturbances using neural networks," Industrial Electronics, IEEE Transactions, vol/issue: 61(5), pp. 2473-2482, 2014.

[17] S. S. Jasper, "Artificial Neural Network Controlled DSTATCOM for Power Quality Improvement," Retrieved, 12th Nov, 2015

[18] Sundaram, Network, Linear Neural, "Power Quality Disturbance Classification Using Adaptive Linear Neural Network (ADALINE) and Feed Forward Neural Network (FFNN)," 2015.

[19] N. N. K. Win, et al., "Comparison Of Power Quality Disturbances Classification Based On Neural Network," International Journal of Scientific \& Technology Research, vol/issue: 4(7), 2015.

[20] D. D. Shakya and S. N. Singh, "SVM based fault location and classification using fuzzy classifier for PQ monitoring," in Power and Energy Society General Meeting-Conversion and Delivery of Electrical Energy in the 21st Century, pp. 1-8, 2008.

[21] W. M. Lin, et al., "Detection and classification of multiple power-quality disturbances with wavelet multiclass SVM," Power Delivery, IEEE Transactions, vol/issue: 23(4), pp. 2575-2582, 2008.

[22] C. Kocaman, et al., "Classification of two common power quality disturbances using wavelet based SVM," in MELECON 2010-2010 15th IEEE Mediterranean Electrotechnical Conference, pp. 587-591, 2010.

[23] D. Taskovski, et al., "Classification of Power Quality Disturbances Using Wavelets and Support Vector Machine," Elektronika ir Elektrotechnika, vol/issue: 19(2), pp. 25-30, 2012.

[24] P. K. Ray, et al., "Optimal feature and decision tree-based classification of power quality disturbances in distributed generation systems,” Sustainable Energy, IEEE Transactions, vol/issue: 5(1), pp. 200-208, 2014. 
[25] N. Sobhan and A. Salemnia, "Detection and Classification of Power-Quality Events Using Discrete Gabor Transform and Support Vector Machine," The 6th International Power Electronics Drive Systems and Technologies Conference, 2015.

[26] H. He and J. Starzyk, "A self-organizing learning array system for power quality classification based on wavelet transform," Power Delivery, IEEE Transactions, vol/issue: 21(1), pp. 286-295, 2006.

[27] S. Suja and J. Jerome, "Power signal disturbance classification using wavelet based neural network," Serbian journal of electrical engineering, vol/issue: 4(1), pp. 71-83, 2007.

[28] B. K. Panigrahi, et al., "A Machine Intelligence Approach for Classification of Power Quality Disturbances," 2009.

[29] H. Shareef and A. Mohamed, "Power quality disturbance detection and visualization utilizing image processing methods," in Proceeding international middle east power conference, Ciro, Egypt, 2010.

[30] S. Nath, et al., "Wavelet based feature extraction for classification of Power Quality Disturbances," Retrieved 20th Nov, 2015.

[31] H. Eristi and Y. Demir, "The feature selection based power quality event classification using wavelet transform and logistic model tree," Przeglad Elektrotechniczny, vol/issue: 88(7a), pp. 43-48, 2012.

[32] S. Roy and S. Nath, "Classification of power quality disturbances using features of signals," International Journal of Scientific and Research Publications, vol/issue: 2(11), 2012.

[33] M. Nayak and S. Dash, "Fuzzy Decision Tree and Particle Swarm Optimization for Mining of Time Series Data," International Journal of Computer Applications, vol/issue: 17(7), pp. 35-41, 2011.

[34] B. Biswal, et al., "A hybrid ant colony optimization technique for power signal pattern classification," Expert Systems with Applications, vol/issue: 38(5), pp. 6368-6375, 2011.

[35] S. Hasheminejad, et al., "Power quality disturbance classification using S-transform and hidden Markov model," Electric Power Components and Systems, vol/issue: 40(10), pp. 1160-1182, 2012.

[36] B. Majhi, et al., "Classification of Power Signals Using PSO based K-Means Algorithm and Fuzzy C Means Algorithm," Journal of Agriculture and Life Sciences, vol/issue: 1(1), 2014.

[37] N. Kumarasabapathy and P. S. Manoharan, "MATLAB Simulation of UPQC for Power Quality Mitigation Using an Ant Colony Based Fuzzy Control Technique," The Scientific World Journal, 2015.

[38] F. Tianrui and O. Sen, "Design of a Distribution Network Power Quality Monitoring System based on Metering Automation Systems and its Application," Indonesian Journal of Electrical Engineering and Computer Science, vol/issue: 10(7), 2012.

[39] J. Jiapei, et al., "A Control Strategy for Single-phase Grid-Connected Inverter with Power Quality Regulatory Function," Indonesian Journal of Electrical Engineering and Computer Science, vol/issue: 12(1), 2014.

[40] F. L. Guo and W. H. Feng, "Application of Grey Correlation Degree and TOPSIS Method in Evaluation of Power Quality," Indonesian Journal of Electrical Engineering and Computer Science, vol/issue: 12(6), 2014.

[41] M. B. I. Reaz, et al., "Expert system for power quality disturbance classifier," Power Delivery, IEEE Transactions, vol/issue: 22(3), pp. 1979-1988, 2007.

[42] C. H. Huang and C. Lin, "Multiple Chaos Synchronization System for Power Quality Classification in a Power System," The Scientific World Journal, 2014.

[43] A. R. T. Góes, et al., "Classification of Power Quality Considering Voltage SAGS in Distribution Systems using KDD Process," Pesquisa Operacional, vol/issue: 35(2), pp. 329-352, 2015.

[44] A. Parizi, et al., "Power Quality Disturbance Classification USING S-Transform and Fuzzy Systems Oriented by PSO Algorithm," International Journal on "Technical and Physical Problems of Engineering, vol/issue: 4(2), pp. 153-161, 2012.

\section{BIOGRAPHIES OF AUTHORS}

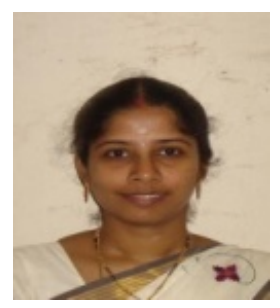

I am B.Devi Vighneshwari, I have completed my BE in EEE and ME in Power System. I have been done my workshop/training on Digital Control of Power Electronic Equipment, CAD forElectrical Drawing, Power System Simulation and Artificial Intelligence and Meta - Heuristic Techniques Applications in Power System.

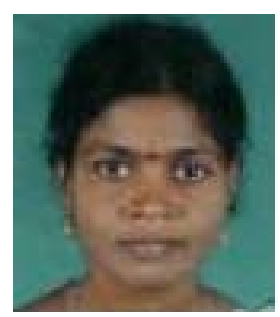

I am Dr. R. Neela Professor of Electrical Engineering, I have completed my B.E in Electrical \& Electronics, M.Tech in Power Systems and PhD in Electrical. I have teching experience is more than 22 years and in Research more than 12 years. 